\title{
Expression and regulation of chemokines in bacterial pneumonia
}

\author{
Theodore J. Standiford, Steven L. Kunkel, " Marc J. Greenberger, Lauri L. Laichalk, \\ and Robert M. Strieter \\ Departments of *Pathology and Medicine, Division of Pulmonary and Critical Care Medicine, The University of \\ Michigan Medical School, Ann Arbor
}

\begin{abstract}
Effective host defense against bacterial invasion is characterized by the vigorous recruitment and activation of inflammatory cells, which is dependent on the coordinated expression of both proand anti-inflammatory cytokines. In this review, we present evidence indicating that both C-X-C and C-C chemokines are integral components of antibacterial host defense. Specifically, in vitro studies indicate that C-X-C chemokines [interleukin-8 (IL-8) and macrophage inflammatory protein 2 (MIP-2)] and the $C-C$ chemokine macrophage inflammatory protein 1 alpha (MIP-1 $\alpha$ ) augment the ability of polymorphonuclear leukocytes (PMNs) and alveolar macrophages, respectively, to phagocytose and kill Escherichia coli. In addition, the intratracheal instillation of Klebsiella pneumoniae in CD-1 mice results in time-dependent production of MIP-2 and MIP-1 $\alpha$, and the inhibition of MIP-2 bioactivity in vivo results in decreases in lung PMN influx, impaired bacterial clearance, and early mortality. Finally, the anti-inflammatory cytokine interleukin-10 (IL-10) is also expressed within the lung during the evolution of Klebsiella pneumonia, and neutralization of IL-10 in vivo results in enhanced proinflammatory cytokine production, bacterial clearance, and increases in both short- and long-term survival. In conclusion, our studies indicate that specific chemokines are important mediators of leukocyte recruitment and/or activation in bacterial pneumonia and that the expression of these chemokines is regulated by endogenously produced IL-10. J. Leukoc. Biol. 59: 24-28; 1996.
\end{abstract}

Key Words: macrophage inflammatory protein $2 \cdot$ macrophage inflammatory protein $1 \alpha \cdot$ interleukin-10 cytokines $\cdot$ Klebsiella $\cdot$ pneumonia $\cdot$ murine

\section{INTRODUCTION}

The lung represents one of the most complex and multifunctional organ systems. The lung not only is involved in the critical process of gas exchange but also is an active and important participant in a variety of immunologic responses. Through its continuous contact with ambient air and enormous vascular bed, the lung is exposed to a large and quite varied burden of foreign antigens, including infectious agents. The lung utilizes a multifaceted system of host defense, which includes mechanical, phagocytic, and immunologic components, to rid itself of injurious substances. The initial barriers to entrance of infectious agents into the airspace include a number of specific mechanical defenses, such as the glottis, the cough reflex, airway secretions, and an intact mucociliary system that lines the entire surface of the large conducting airways [1]. When defense mechanisms of the conducting airways are breached, the phagocytic system of the terminal airspaces becomes critical to eliminating injurious agents from the lung. The alveolar macrophage is the primary resident phagocytic cell of the alveolus. In the setting of a low bacterial burden or exposure to less virulent gram-positive organisms, the alveolar macrophage can effectively phagocytose and kill invading organisms [1, 2]. However, when the bacterial burden is large or when more virulent gramnegative organisms, such as Pseudomonas aeruginosa or Klebsiella pneumoniae, gain assess to the lower airspaces, the recruitment of neutrophils (PMNs) is essential for effective containment and clearance of bacteria. In addition, specific bacterial infections, including infections with $K$. pneumoniae, are associated with an appreciable infux of blood mononuclear phagocytes, although the contribution of these cells to bacterial clearance is not known [3].

\section{CYTOKINES INVOLVED IN ANTIBACTERIAL HOST DEFENSE}

The generation of inflammation in the setting of bacterial challenge is a complex and dynamic process that involves the coordinated expression of both pro- and anti-inflamma-

\footnotetext{
Abbreviations: AM, alveolar macrophage; BALF, bronchoalveolar lavage fluid; CFU, colony-forming unit; IL-10, interleukin-10; MCP-1, monocyte chemotactic protein 1; MIP-2, macrophage inflammatory protein 2; MPO, myeloperoxidase; PMN, polymorphonuclear leukocyte; TNF- $\alpha$, tumor necrosis factor $\alpha$.

Reprint requests: Theodore J. Standiford, Department of Internal Medicine, Division of Pulmonary and Critical Care Medicine, The University of Michigan Medical Center, 3916 Taubman Center, Box 0360, Ann Arbor, MI 48109-0360.

Received September 1, 1995; accepted September 26, 1995.
} 
tory cytokines. Several cytokine mediators, including tumor necrosis factor-alpha (TNF- $\alpha$ ), interferon-gamma, and granulocyte colony-stimulating factor, have been shown to play important roles in host defense against bacterial organisms [4-6]. Two closely related families of chemotactic cytokines, the $\mathrm{C}-\mathrm{X}-\mathrm{C}$ and the $\mathrm{C}-\mathrm{C}$ chemokine families, have been increasingly recognized as important mediators in a variety of inflammatory disease states [7]. The C-X-C family, which includes interleukin-8 (IL-8), macrophage inflammatory protein 2 (MIP-2), GR0, ENA-78, and NAP2, has predominant PMN stimulatory and chemotactic activities, and the C-C family, which includes monocyte chemotactic protein 1 (MCP-1), MCP-2, MCP-3, RANTES, MIP- $1 \alpha$, and MIP-1 $\beta$, exerts predominant macrophage activating and chemotactic effects. The roles of C-X-C or C-C chemokines in mediating leukocyte activation and/or recruitment in bacterial pneumonia have not been characterized.

\section{POTENTIAL ROLE OF CHEMOKINES IN BACTERIAL PNEUMONIA}

Several lines of evidence suggest that C-X-C and C-C chemokines may be critically involved in host defense against infection, particularly in response to bacterial organisms. The previously described leukocyte stimulatory and chemotactic properties attributable to chemokines would predict that these molecules may enhance the microbicidal functions of PMNs and macrophages [7]. In addition, a number of infectious agents, or cell wall components of these agents, have been shown to induce the production of C-X-C or C-C chemokines from mononuclear phagocytes and stromal cells. Specifically, bacterial products including lipopolysaccharide, lipoteichoic acid, capsular polysaccharides, and Mycobacterium tuberculosis purified protein derivative stimulate the production of IL8, MCP-1, or MIP-1 $\alpha$ from mononuclear phagocytes [7-11]. Moreover, the movement of invasive bacterial species through either epithelial or endothelial monolayers can trigger IL-8 release from these cells [12]. Finally, the phagocytosis of microbes, such as $M$. tuberculosis, can serve as a signal for the secretion of chemokines, including IL-8 and MCP-1 [13].

Additional indirect evidence supporting a role of chemokines in infection stems from the observation that chemokines, in particular IL-8, have been detected in increased amounts within the sputum or bronchoalveolar lavage fluid (BALF) obtained from patients with acute pulmonary infection. Rodriguez and colleagues [14] found an increased incidence of bacterial pneumonia in trauma patients who had IL-8 present in bronchial secretions. In addition, antigenic IL-8 has been detected in BALF of patients with Pneumocystis carinii pneumonia, and levels of IL-8 were closely correlated with the clinical severity of disease [15]. Finally, we have detected increased amounts of IL-8 mRNA and protein levels in the sputum and bronchoalveolar lavage fluid of patients with cystic fibrosis in exacerbation (unpublished observations). Interleukin-8 accounted for approximately $40-50 \%$ of the increased PMN chemotactic activity found in the BALF obtained from these patients during exacerbation of disease. In addition, IL-8 levels were closely correlated with numbers of PMNs in BALF and with disease activity as assessed by several clinical parameters. Interestingly, a dramatic decline in the expression of IL-8 mRNA from BALF cells occurred after treatment with antibiotics with or without corticosteroids. Although finding increased amounts of chemokines expressed during bacterial infection does not confirm a cause-and-effect relationship, it does support a possible role for these chemokines as critical mediators in bacterial infection, as well as potentially useful diagnostic and prognostic indicators in these patients.

\section{EFFECT OF CHEMOKINES ON LEUKOCYTE PHAGOCYTIC AND BACTERICIDAL ACTIVITY IN VITRO}

Effective clearance of microbes by leukocytes (both PMNs and mononuclear phagocytes) is dependent on initial attachment, phagocytosis, and eventual intracellular killing $[16,17]$. The efficacy of phagocytosis is chiefly determined by the presence of specific (immunoglobulin) and/or nonspecific (complement) opsonins on the surface of microbes, as well as the expression of specific complement or immunoglobulin receptors on the surface of leukocytes [16]. The effect of chemokines on leukocyte phagocytic activity has been incompletely characterized. Hostoffer and colleagues [18] observed that IL-8 did not alter immunoglobulin A-mediated phagocytosis of $P$. aeruginosa by PMNs, although IL-8 did appear to increase the cell surface expression of C3 receptor on PMNs. In contrast, studies in our laboratory indicate that treatment of human PMNs with either human recombinant (hr) IL-8 or murine recombinant (mr) MIP-2 (the likely functional murine homologue of IL-8) for $1 \mathrm{~h}$ in the presence of $10 \%$ human serum resulted in an approximately 1.6- and 2.1-fold increase in phagocytosis of Escherichia coli, respectively, compared with PMNs incubated in serum alone (data not shown). These effects could not be completely explained by changes in leukocyte complement or immunoglobulin receptor expression, as incubation of PMNs with either IL-8 or MIP-2 resulted in only modest increases in PMN C3 receptor expression, with no significant change in Fc $\gamma$ II or FcyIII receptor expression.

The effect of chemokines on leukocyte bactericidal and fungicidal activity has been studied in somewhat more detail. Interleukin-8 has been shown to enhance PMN microbicidal activity against Mycobacterium fortuitum and PMN fungistatic activity against Candida albicans $[19,20]$. Similarly, we have observed that hrIL-8 and mrMIP-2 enhanced the ability of PMN to kill intracellular $E$. coli in a dose-dependent fashion, resulting in a maximal 4- and 5.6-fold increase in killing, respectively, compared with untreated PMNs (Table 1). Leukocyte microbicidal activ- 
TABLE 1. Leukocyte Bactericidal Activity ${ }^{a}$

\begin{tabular}{lll}
\hline \hline Cells & Treatment & E. coli CFU \\
\hline PMN & Control & $21.9 \pm 0.12 \times 10^{3}$ \\
& muMIP-2 $(10 \mathrm{ng} / \mathrm{ml})$ & $4.26 \pm 0.11 \times 10^{3 *}$ \\
& hull-8 $(10 \mathrm{ng} / \mathrm{ml})$ & $5.49 \pm 0.13 \times 10^{3 *}$ \\
AM & Control & $12.6 \pm 0.11 \times 10^{2}$ \\
& muMIP-1 $\alpha(10 \mathrm{ng} / \mathrm{ml})$ & $6.16 \pm 0.11 \times 10^{2 *}$ \\
& muTNF $(10 \mathrm{ng} / \mathrm{ml})$ & $4.57 \pm 0.14 \times 10^{2 *}$ \\
\hline
\end{tabular}

Becterial killing of phagocytoeed $E$. coli by resting and stimulated human PMNs or murine AMs. Resting or cytokine-stimulated PMN or AM $\left(1 \times 10^{6}\right)$ were incubated with $10 \%$ human serum and $E$. coli $\left(1 \times 10^{7}\right)$ in HBSS for $1 \mathrm{~h}$; then cell lysate CFU was determined. Values represent mean I SEM of 12 separate experiments. ${ }^{*} P<.01$ compared with unstimulated control.

ity can occur by oxidative or nonoxidative pathways. The mechanism of chemokine-induced PMN bacterial killing has not yet been fully elucidated. However, nonoxidative rather than oxidative events are more likely involved, as C-X-C chemokines such as IL-8 and MIP-2 are more potent inducers of PMN degranulation than the respiratory burst $[7,21,22]$ and IL-8-induced PMN microbicidal activity has previously been shown to be unaffected by coincubation with antioxidants [19].

Just as C-X-C chemokines regulate PMN phagocytic and microbicidal activities, C-C chemokines can enhance the antimicrobial effects of mononuclear phagocytes. Nakano and associates [23] have demonstrated that hrMCP-l greatly augmented the phagocytosis and killing of $P$. aeruginosa and Salmonella typhimurium by murine peritoneal macrophages. Similarly, we have observed that treatment of murine alveolar macrophages with mrMIP-l $\alpha$ resulted in both an increase in phagocytosis (data not shown) and an approximately twofold increase in $E$. coli killing, as compared with unstimulated alveolar macrophages (Table 1). Collectively, these studies indicate that members of both the C-X-C and C-C chemokine families can significantly enhance the ability of the primary phagocytic cells of the lung (PMNs and alveolar macrophages) to phagocytose and kill $E$. coli.

\section{ROLE OF CHEMOKINES IN MURINE MODELS OF BACTERIAL PNEUMONIA}

The relative contribution of chemokines to effective containment and eventual clearance of infectious agents from the lung in vivo has not previously been defined. In related studies by others, administration of hrIL-8 to nonneutropenic mice challenged with $K$. pneumoniae intraperitoneally (i.p.) was protective, whereas IL-8 treatment of neutropenic mice administered $K$. pneumoniae or mice (neutropenic and nonneutropenic) challenged with $P$. aeruginosa resulted in no change in mortality or, in some instances, increased mortality [24]. In murine models of $P$. aeruginosa or $S$. typhimurium peritonitis, the i.p. administration of hrMCP-l $(2.5 \mu \mathrm{g} /$ animal) resulted in complete protection from lethality [23]. To begin to address the role of specific chemokines in lung antibacterial host defense, we have developed a murine model of Klebsiella pneumo- nia. In this model, CD-1 mice were intratracheally inoculated with $10^{3}$ colony-forming units (CFU) of $K$. pneumoniae, strain 43816, serotype 2 (ATTC, Rockville, MD). This strain was used in our experiments because this organism is a heavily encapsulated species that is particularly virulent in mice [25]. Histologic examination of the lungs $48 \mathrm{~h}$ after the intratracheal (i.t.) inoculation with $K$. pneumoniae revealed substantial accumulation of $\mathrm{PMNs}$ and moderate numbers of macrophages within the lung airspace and interstitium. Temporally correlated with the development of pulmonary inflammation was the production of TNF- $\alpha$, MIP-2, and MIP-1 $\alpha$. As shown in Figure 1 , a significant time-dependent increase in the levels of these cytokines was noted in lung homogenates after the i.t. administration of $K$. pneumoniae, with maximal 13-, 14-, and 8-fold increases in lung homogenate TNF- $\alpha$, MIP-2, and MIP-1 $\alpha$ levels observed, respectively, compared with saline-treated control animals $(P<.05$ for all cytokines). Immunohistochemical analysis indicated that the alveolar macrophage was a major cellular source of these cytokines (data not shown). However, recruited PMNs also appear to express cell-associated TNF- $\alpha$ and MIP-2.

To determine the biologic relevance of MIP-2 to PMN influx in Klebsiella pneumonia, animals were passively immunized with either rabbit preimmune serum or rabbit anti-murine MIP-2 antibody i.p. $2 \mathrm{~h}$ prior to the i.t. administration of $K$. pneumoniae (or saline control); then lungs

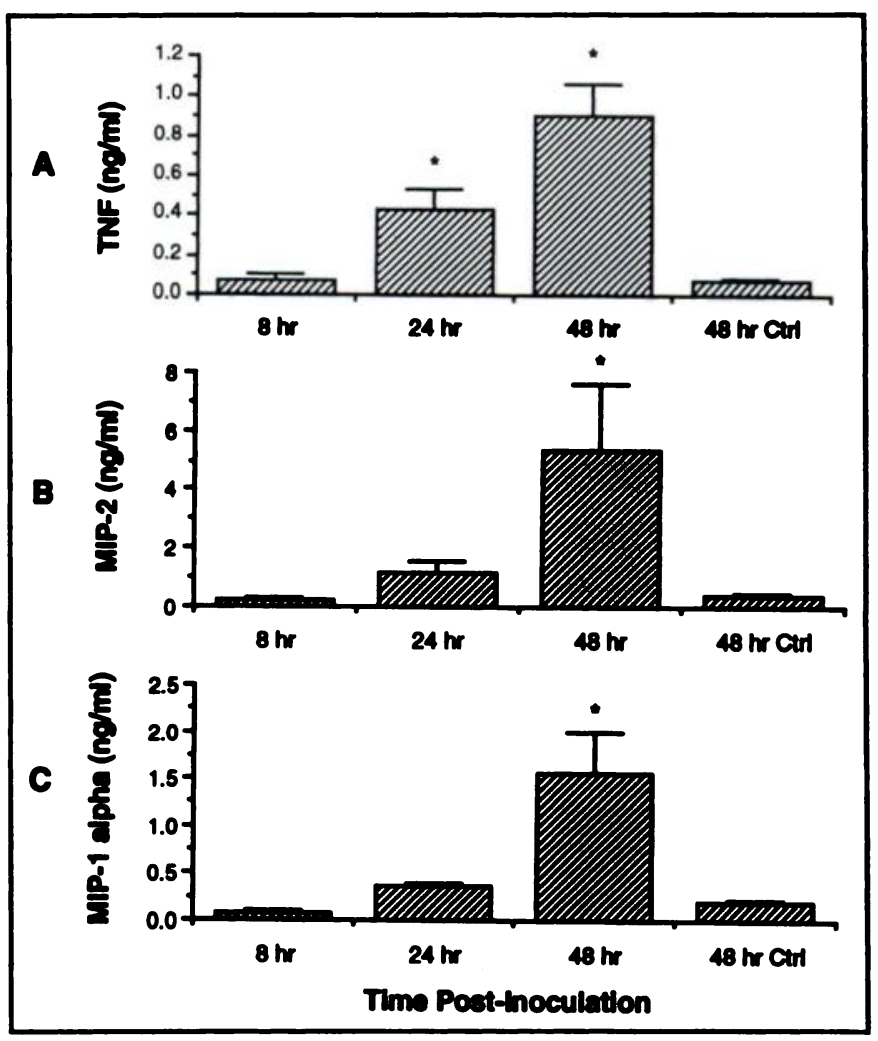

Fig. 1. Time-dependent production of MIP-2 and MIP- $1 \alpha$ protein in lung homogenates after the i.t. administration of saline or $K$. pneumoniae $\left(10^{3}\right.$ CFU). ${ }^{*} P<.05$ compared with $48 \mathrm{~h}$ control. Experimental $n=5$ per group. 
were removed $48 \mathrm{~h}$ later and assayed for myeloperoxidase (MPO) activity as a measure of PMN influx. Lung MPO activity in anti-MIP-2-treated animals inoculated with Klebsiella was decreased by $60 \%$ at $48 \mathrm{~h}$, compared with animals receiving preimmune serum $(P<.05$, data not shown). In addition, treatment with anti-MIP-2 antibodies resulted in a 5.8- and 4.4-fold increase in viable $K$. pneumoniae in the lungs at 24 and $48 \mathrm{~h}$ postinoculation, respectively, compared with animals receiving preimmune serum (Table 2). Furthermore, inhibition of MIP-2 bioactivity in vivo resulted in early dissemination of $K$. pneumoniae to both blood and liver (Table 2). Finally, as compared with animals receiving preimmune serum, passive immunization with anti-MIP-2 serum resulted in a $40-50 \%$ increase in early $(24-48 \mathrm{~h})$ but not late $(>72 \mathrm{~h})$ mortality (data not shown). These studies indicate that MIP-2 is an important mediator of PMN influx and effective bacterial clearance in murine Klebsiella pneumonia. The contribution of MIP-l $\alpha$ to lung antibacterial host defense in Klebsiella pneumonia is the focus of ongoing studies.

\section{ROLE OF IL-10 IN ACUTE BACTERIAL PNEUMONIA}

The generation of inflammation is critical to effective clearance of microbial agients. However, the control of lung inflammatory responses is equally important to preventing excessive leukocyte-mediated tissue injury. Interleukin-10 (IL-10) is a cytokine that was initially identified as an important mediator of TH2-driven immune responses [26]. This cytokine has been shown to exert anti-inflammatory properties, in part by down-regulating the expression of TNF, IL-1, and members of both the C-X-C and C-C chemokine families $[27,28]$. We have shown that endogenously produced IL-10 plays an important role in damping the overzealous production of cytokines in endotoxemia, as the passive immunization of mice with anti-IL-10 followed by challenge with sublethal doses of lipopolysaccharide $(100 \mu \mathrm{g})$ i.p. results in a marked increase in the magnitude of TNF- $\alpha$ and MIP-2 expression, enhanced lung injury, and substantial increases in mortality [29]. Furthermore, administration of IL-10 to mice has

TABLE 2. K. preumoniae CFU in Plasma and Liver $24 \mathrm{~h}$ After Inoculation $^{a}$

\begin{tabular}{llc}
\hline \hline Site & Treatment & K. pneumoniae CFU \\
\hline Placma & Preimmune & $1.17 \pm 0.21 \times 10^{1}$ \\
& Anti-MIP-2 & $2.82 \pm 0.45 \times 10^{1}$ \\
Liver & Preimmune & $3.47 \pm 0.35 \times 10^{1}$ \\
& Anti-MIP-2 & $11.2 \pm 0.18 \times 10^{1 *}$ \\
Lung & Preimmune & $1.74 \pm 0.10 \times 10^{3}$ \\
& Anti-MIP-2 & $10.1 \pm 0.40 \times 10^{3 *}$ \\
\hline
\end{tabular}

-Effect of anti-MIP-2 serum on $K$. pnewmoniae CFU in plasma, liver, and lung at $24 \mathrm{~h}$ after inoculation. Animals were pretreated with either $0.5 \mathrm{ml}$ of rabbit anti-MIP-2 antibody or rabbit preimmune serum i.p. 2 h prior to i.t. inoculation.

$* P<.05$ compared with animals receiving preimmune serum.
TABLE 3. $K$. pneumoniae CFU in Plasma and Lung ${ }^{a}$

\begin{tabular}{lcc}
\hline \hline Site & Treatment & K. pneumoniae CFU \\
\hline Plasma & Preimmune & $245 \pm 0.32 \times 10^{3}$ \\
& Anti-IL-10 & $1.82 \pm 0.43 \times 10^{3 * *}$ \\
Lung & Preimmune & $15.5 \pm 0.18 \times 10^{7}$ \\
& Anti-IL-10 & $4.76 \pm 0.18 \times 10^{7 *}$ \\
\hline
\end{tabular}

-Effect of anti-IL-10 serum on $K$. pneumoniae CFU in plasma and lung $48 \mathrm{~h}$ after inoculation. Animals were pretreated with either $0.5 \mathrm{ml}$ of rabbit anti-IL-10 antibody or rabbit preimmune serum i.p. $2 \mathrm{~h}$ prior to i.t. inoculation.

$* * P<.01, * P<.05$ compared with animals receiving preimmune serum.

been shown by others to markedly attenuate endotoxin-induced lethality [30]. However, IL-10-mediated suppression of activating and chemotactic cytokines may be detrimental to the host in the setting of infectious pneumonia, where a vigorous inflammatory response is essential for effective microbial clearance. In support of this premise, IL-10 inhibits macrophage antimicrobial activity in vitro [31]. In addition, the exaggerated expression of IL-10 in patients with leprosy has been associated with persistent and chronic infection [32], and the inhibition of IL-10 bioactivity in vivo promotes effective clearance of $\mathrm{Myco}$ bacterium avium-intracellulare in mice [33].

To test the hypothesis that IL-10 may have detrimental effects on lung antibacterial host defense, we first assessed the effect of mrIL-10 on PMN phagocytic activity. Treatment of PMNs with IL-10 resulted in a 33\% reduction of phagocytosis of $E$. coli and a maximal 3.9-fold decrease in killing of intracellular $E$. coli, as compared with unstimulated PMNs (data not shown). We next assessed the contribution of IL-10 to effective bacterial clearance of $K$. pneumoniae in vivo. Interestingly, inoculation of animals with $K$. pneumoniae resulted in time-dependent production of both IL-10 mRNA and protein, with maximal expression occurring at $\mathbf{4 8} \mathrm{h}$ after inoculation (data not shown). To further define the biologic role of endogenously produced IL-10 in Klebsiella pneumonia, animals were pretreated with rabbit anti-murine IL-10 serum $2 \mathrm{~h}$ prior to inoculation with $K$. pneumoniae. Treatment with anti-IL-10 antibodies resulted in 3.6-, 3.0-, and 1.8-fold increases in TNF- $\alpha$, MIP-2, and MIP-1 $\alpha$ protein levels in lung homo-

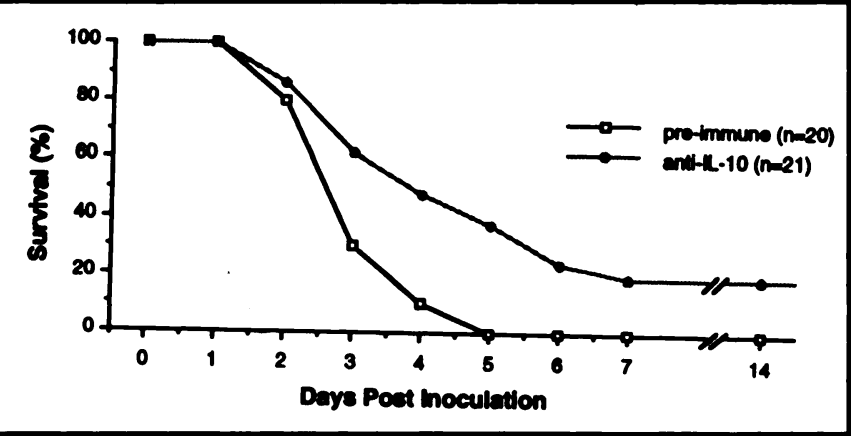

Fig. 2. Effect of anti-IL-10 serum on survival in Klebsiella pneumonia. CD-l mice were pässively immunized with $0.5 \mathrm{ml}$ of rabbit preimmune or rabbit anti-murine IL-10 serum 2 h prior to inoculation with $K$. pneumoniae (10 $\mathrm{CFU})$. 
genates at $48 \mathrm{~h}$, as compared with animals receiving preimmune serum. Furthermore, passive immunization with anti-IL-10 antibodies resulted in an 3.3-fold reduction in lung $K$. pneumoniae CFU and a greater than 100 -fold decrease in numbers of $K$. pneumoniae cultured from blood at $48 \mathrm{~h}$ (Table 3). Survival of anti-IL-10-treated animals inoculated with $K$. pneumoniae was $40 \%$ at 5 days, with $20 \%$ of animals surviving past 14 days, whereas no animals in the control group survived to 5 days (Fig. 2).

In summary, our studies indicate that (1) the chemokines MIP-2 and MIP-1 $\alpha$ stimulate PMN and AM phagocytic and bactericidal activity in vitro, respectively; (2) MIP-2 and MIP-1 $\alpha$ are produced during the evolution of Klebsiella pneumonia; (3) the inhibition of MIP-2 bioactivity in vivo results in decreased PMN influx and bacterial clearance, and increased early but not late mortality; and (4) the inhibition of IL-10 bioactivity in animals with Klebsiella pneumonia results in increased expression of TNF- $\alpha$, MIP-2, and MIP-1 $\alpha$, enhanced bacterial clearance, and prolonged survival. We conclude that specific chemokines are important mediators of leukocyte recruitment and/or activation in bacterial pneumonia and that the expression of these chemokines is regulated by endogenously produced IL-10. Immunologic manipulation of pro- and/or anti-inflammatory cytokine expression may serve as an important adjuvant therapy in the treatment of immunocompromised and immunocompetent patients with severe bacterial pneumonia.

\section{ACKNOWLEDGMENTS}

This research was supported in part by National Institutes of Health grants 1P50HL46487, HL50057, HL02401, HL31693, HL35276, HL52800, and AA10571.

\section{REFERENCES}

1. Nelson, S., Mason, C.M., Knolls, J., Summer, W.R. (1995) Pathophysiology of pneumonia. Clin. Chest Med. 16, 1-12.

2. Onofrio, J.M., Toews, G.B., Lipscomb, M.F. (1983) Granulocyte-alveolar macrophage interaction in the pulmonary clearance of Staphylococcus aureus. Am. Rev. Respir. Dis. 127, 335-342.

3. Toews, G.B., Gross, G.N., Pierce, A.K. (1979) The relationship of inoculum size to lung bacterial clearance and phagocytic cell response in mice. Am. Rev. Respir. Dis. 120, 559-566.

4. Nakano, Y., Onozuka, K., Yerada, Y., Shinomiya, H., Nakano, M. (1990) Protective effect of recombinant tumor necrosis factor- $\alpha$ in murine salmonellosis. J. Immunol. 144, 1935-1941.

5. Kolls, J.K., Nelson, S., Summer, W.R. (1993) Recombinant cytokines and pulmonary host defense. Am. J. Med. Sci. 306, 330-335.

6. Nelson, S. (1991) Overview of granulocyte colony-stimulating factor in bacterial infections in the non-neutropenic host. Clin. Infect. Dis. 143S, $398-410$.

7. Oppenheim, J.J., Zachariae, C.O.C., Mukaida, N., Matsushima, K. (1991) Properties of the novel proinflammatory supergene intercrine cytokine family. Annu. Rev. Immunol. 9, 617-647.

8. Standiford, T.J., Arenberg, D.A., Danforth, J.M., Kunkel, S.L., VanOtteren, G.M., Strieter, R.M. (1994) Lipotheichoic acid induces the secretion of interleukin-8 from human blood monocytes: a cellular and molecular analysis. Infect. Immun. 62, 119-125.

9. Danforth, J.M., Strieter, R.M., Arenberg, D.A., Kunkel, S.L., VanOtteren, G.M., Standiford, T.J. (1995) Induction of MIP-la in-vitro and in-vivo: the role of lipoteichoic acid. J. Clin. Immunol. Immunopathol. 74, 77-83.

10. Soell, M., Diab, M., Haan-Archipoff, G., Beretz, A., Herbelin, C., Poutrel, B., Klein, J. (1995) Capsular polysaccharide types 5 and 8 of Staphylococcus aureus bind specifically to human epithelial (KB) cells, endothelial cells, and monocytes and induces release of cytokines. Infect. Immun. 63, 1380-1386.

11. Friedland, J.S., Shattock, R.J., Griffin, G.E. (1993) Phagocytosis of Mycobacterium tuberculosis or particulate stimuli by human monocytic cells induces equivalent monocyte chemotactic protein-l gene expression. Cytokine 5, 150-156.

12. Kasahara, K., Tobe, T., Tomita, M., Mukaida, N., Shao-Bo, S., Matsushima, K., Yoshida, T., Sugihara, S., Kobayahi, K. (1994) Selective expression of monocyte chemotactic and activating factor/monocyte chemoattractant protein 1 in human blood monocytes by Mycobacterium tuberculosis. J. Infect. Dis. 170, 1238-1247.

13. Eckmann, L., Kagnoff, M.F., Fierer, J. (1993) Epithelial cells secrete the chemokine interleukin-8 in response to bacterial entry. Infect. Immun. 61, $4569-4574$.

14. Rodriguez, J.L., Miller, C.G., DeForge, L.E., Kelty, L., Shanley, C.J., Bartlett, R.H., Remick, D. (1992) Local production of interleukin-8 is associated with nosocomial pneumonia. J. Trauma 33, 74-81.

15. Benfield, T.L., Vestbo, J., Junge, J., Nielson, T.L., Jenson, A.B., Lundgren, J.D. (1995) Prognostic value of interleukin-8 in AIDS-associated Pneumocystis carinii pneumonia. Am. J. Respir. Crit. Care Med. 151, 1058-1062.

16. Horwitz, M.A. (1982) Phagocytosis of microorganisms. Rev. Infect. Dis. 4, 104-123.

17. Lehrer, R.I., Ganz, T. (1990) Antimicrobial polypeptides of human neutrophils. Blood 76, 2169-2181.

18. Hostoffer, R.W., Krukovets, I., Berger, M. (1994) Enhancement by tumor necrosis factor- $\alpha$ of Fca receptor expression and IgA-mediated superoxide generation and killing of Pseudomonas aeruginosa by polymorphonuclear leukocytes. J. Infect. Dis. 170, 82-87.

19. Nibbering, P.H., Pos, O., Stevenhagen, A., Van Furth, R. (1993) Interleukin8 enhances nonoxidative intracellular killing of Mycobacterium fortuitum by human granulocytes. Infect. Immun. 61, 3111-3116.

20. Djeu, J.Y., Matsushima, K., Oppenheim, J.J., Shiotsuki, K., Blanchard, D.K. (1990) Functional activation of human neutrophils by recombinant monocyte-derived neutrophil chemotactic factor/IL-8. J. Immunol. 144, 2205-2210.

21. Wolpe, S.D., Sherry, B., Juers, D., Davatelis, G., Yurt, R.W., Cerami, A. (1989) Identification and characterization of macrophage inflammatory protein 2. Proc. Natl. Acad. Sci. USA 86, 612-616.

22. Wolpe, S.D., Cerami, A. (1989) Macrophage inflammatory proteins 1 and 2: members of a novel superfamily of cytokines. FASEB J. 3, 2565-2573.

23. Nakano, Y., Kasahara, T., Mukaida, N., Ko, Y., Nakano, M., Matsushima, K. (1994) Protection against lethal bacterial infection in mice by monocytechemotactic and -activating factor. Infect. Immun. 62, 377-383.

24. Vogels, M.T.E., Lindley, I.J.D., Curfs, J.H.A.J., Eling, W.M.C., van der Meer, J.W.M. (1993) Effects of interleukin-8 on nonspecific resistance to infection in neutropenic and normal mice. Antimicrob. Agents Chemoeher. 37, 276-280.

25. Bakker-Woudenberg, I.A.J.M., Lokerse, A.F., ten Kate, M.T., Mouton, J.M., Woodle, M.C., Storm, G. (1993) Liposomes with prolonged blood circulation and selective localization in Klebsiella pneumoniae-infected lung tissue. J. Infect. Dis. 168, 164-171.

26. Howard, M., O'Garra, A., Ishida, H., Malefyt, R.D.W., De Vries, J. (1992) Biological properties of interleukin 10. J. Clin. Immunol. 12, 239-247.

27. Fiorentino, D.F., Zlotnik, A., Mosmann, T.R., Howard, M., O'Garra, A. (1991) IL-10 inhibits cytokine production by activated macrophages. J. Immunol. 147, 3815-3822.

28. Kasama, T., Strieter, R.M., Lukacs, N.W., Burdick, M.D., Kunkel, S.L. (1994) Regulation of neutrophil-derived chemokine expression by IL-10. J. Immunol. 152, 3559-3569.

29. Standiford, T.J., Strieter, R.M., Lukacs, N.W., Kunkel, S.L. (1995) Neutralization of IL-10 increases lethality in endotoxemia: cooperative effects of macrophage inflammatory protein-2 and tumor necrosis factor. J. Immunol. $155,2222-2229$.

30. Howard, M., Muchamuel, T., Andrade, S., Menon, S. (1993) Interleukin 10 protects mice from lethal endotoxemia. J. Exp. Med. 177, 1205-1208.

31. Oswald, I.P., Wynn, T.A., Sher, A., James, S.L. (1992) Interleukin 10 inhibits macrophage microbicidal activity by blocking endogenous production of tumor necrosis factor $\alpha$ required as a costimulatory factor for interferon $\gamma$-induced activation. Proc. Natl. Acad. Sci. USA 89, 8676-8680.

32. Sieling, P.A., Abrams, J.S., Yamamura, M., Salgame, P., Bloom, B.R., Rea, T.H., Modlin, R.L. (1993) Immunosuppressive roles for IL-10 and IL-4 in human infection: in vitro modulation of $\mathrm{T}$ cell responses in leprosy. $J$. Immunol. 150, 5501-5510.

33. Bermudez, L.E., Champsi, J. (1993) Infection with Mycobaterium avium induces production of interleukin-10 (IL-10), and administration of anti-IL 10 antibody is associated with enhanced resistance to infection in mice. Infect. Immun. 61, 3093-3097. 